International Mathematical Forum, 2, 2007, no. 66, 3273 - 3277

\title{
Zero Gravity of Free-Surface Jet Flow
}

\author{
B. Bouderah ${ }^{1}$, A. Gasmi and H. Serguine \\ Laboratory of Pure and Applied Mathematics LPAM \\ University of M'sila, (28000) Algeria
}

\begin{abstract}
Our aim in this paper, is the studies of flow due to a jet against a infinite vertical plate on the free surface, where the effects of gravity and surface tension is not taken into account. We use initially the method of the free streamline theory based on the hodograph method and Schwarz-Christoffel transformation technique to obtain the exact solution.
\end{abstract}

Mathematics Subject Classification: 76B10, 76C05, 76M45

Keywords: Free surface, Flow, Jet, Streamline theory

\section{Introduction}

The problem of determining the free surface due to a jet against a vertical plate is considered. The classical problem of free streamline flow of an ideal fluid has been studied by many authors $[1-4]$. The first work in this type of problem is characterized by the use of the Schwartz-Christoffel formula. The latter can treat the flows of border, which combines rectilinear paroies and a free surface. A two-dimensional flow of a jet of a ideal fluid encroaching on a wall neglecting the forces of gravity studied by W. Peng and D. F. Parker [1] using the integral equations method.

\section{Formulation of the problem}

The fluid is assumed to be inviscid, incompressible and irrotationnal. The effects of gravity and surface tension are not taken into account. Since the flow is symmetrical, one can then study the problem only on the higher plane half. One takes as locates co-ordinates, the thread of current $\mathrm{AB}$ on $x$-axis and wall $\mathrm{BC}$ on the $y$-axis (see Figure 1.).

\footnotetext{
${ }^{1}$ bouderah_b@yahoo.fr
} 


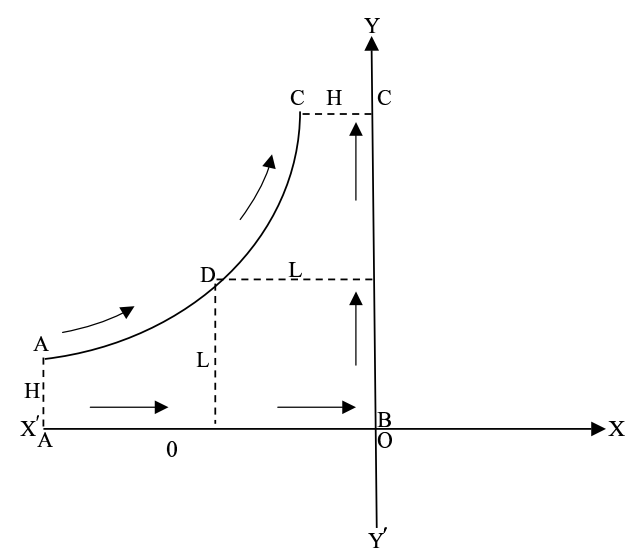

Figure 1: The $f$-plan

It is supposed that the flow is uniform speed $\mathrm{U}$ and of amplitude $\mathrm{H}$, when $x \rightarrow-\infty$ and $y \rightarrow+\infty$. The flow is limited superiorly by the thread of current $\mathrm{AC}$, for $x$ negative. One indicates by $\xi=u-i v$ the complex velocity such as $u$ and $v$ are the components of the velocity and by $f=\phi+i \psi$ the complex potential function where $\phi$ and $\psi$ be the potential and the stream functions respectively. The function $f$ transforms $z$-plan into an infinite band. (see Figure 2.)

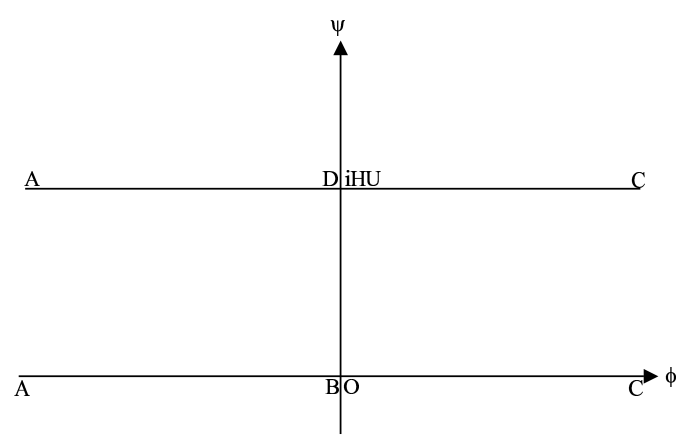

Figure 2: The $z$-plan

The mathematical problem consists in determining the potential function $\phi$ which checks the following conditions: 


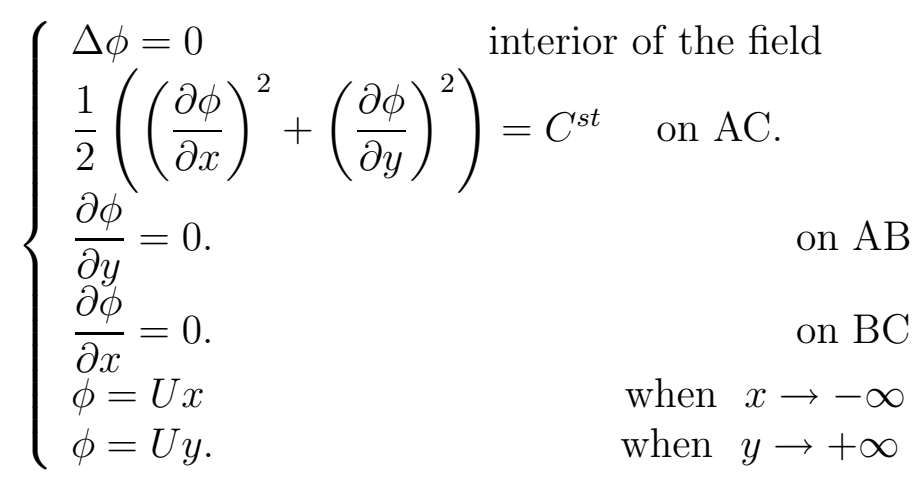

\section{Resolution of the problem}

To solve this problem, we use initially the method of the free streamline theory introduced by Kirchoff, based on the hodograph transformation . The complex transformation is defined:

$$
\Gamma=\log \left(\frac{U d z}{d f}\right)=\log \left(\frac{U}{u-i v}\right)=\log \left(\frac{U}{q}\right)+i \theta .
$$

Where $z=x+i y, q$ and $\theta$ are the module speed and the angle between the velocity vector and the horizontal one, respectively. By this last transformation, the field occupied by the fluid in the $z$-plan is transformed into a semi-infinite band in the $\Gamma$-plan .(see Figure 3.)

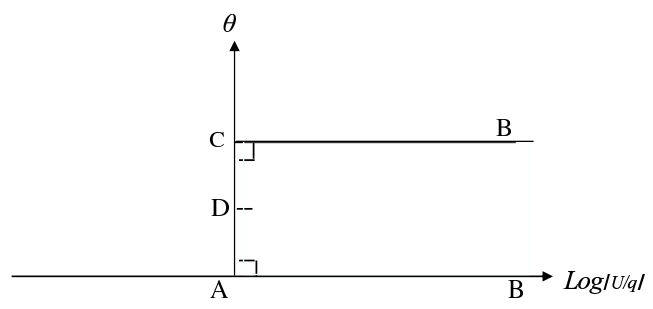

Figure 3: The $\Gamma-$ plan

The conforms transformation of an semi-infinite band in the plan to the lower half-plan of another complexes $\beta$-plan, is given by the theorem of Schwartz-Christoffel, by respecting the direction and the orientation of the flow.(see Figure 4.)

This transformation is given by:

$$
\beta=\frac{1}{2}[1-\cosh (2 \Gamma)]=-\sinh ^{2} \Gamma
$$




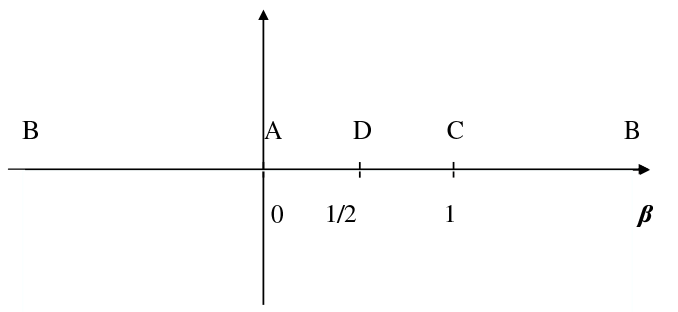

Figure 4: The $\Gamma-$ plan

The transformation which transforms the interior of the infinite band of the $f$-plan towards the lower half-plan of the $\beta$-plan is:

$$
\beta=\frac{1}{1-\exp \left(\frac{\pi f}{H U}\right)}
$$

After calculations, we finds a relation between $\beta$ and $z$ :

$$
U \frac{d z}{d \beta}=U \frac{d z}{d f} \frac{d f}{d \beta}=-\frac{H U}{\pi}\left[\frac{1}{\beta \sqrt{1-\beta}}+i \frac{1}{(1-\beta) \sqrt{\beta}}\right]
$$

By integrating (5) with the choice of $z_{0}=-L+i L$ into the point $\mathrm{D}$ where $\beta=1 / 2$. The solution is as follows:

$$
\left\{\begin{array}{cl}
x=-L-\frac{H}{\pi} \log \left(\frac{1+\sqrt{1-\beta}}{1-\sqrt{1-\beta}}\right)+\frac{2 H}{\pi} \log (\sqrt{2}+1) . & \\
y=L+\frac{H}{\pi} \log \left(\frac{1+\sqrt{\beta}}{1-\sqrt{\beta}}\right)-\frac{2 H}{\pi} \log (\sqrt{2}+1) . & 0 \leq 1
\end{array}\right.
$$

The amplitude of the jet in the origine is:

$$
H=\lim _{\beta \longrightarrow 0} y(\beta)=L-\frac{2 H}{\pi} \log (\sqrt{2}+1) .
$$

One represents $C$ like the contraction degree of the flow:

$$
C=\frac{H}{L}=0.64
$$
becomes:

We write: $\bar{x}=\frac{x}{L} \cdot \bar{y}=\frac{y}{L}$, the parametric equation of the free surface 


$$
\left\{\begin{array}{cc}
\bar{x}=-1-\frac{1}{\pi} \log \left(\frac{1+\sqrt{1-\beta}}{1-\sqrt{1-\beta}}\right) . & \\
\bar{y}=1+\frac{1}{\pi} \log \left(\frac{1+\sqrt{\beta}}{1-\sqrt{\beta}}\right) . & 0 \leq \beta \leq 1
\end{array}\right.
$$

We takes the symmetry of the curve which represents the equation (10) compared to the $x$-axis, for determining the form of the free surface of the jet. (see Figure 5.)

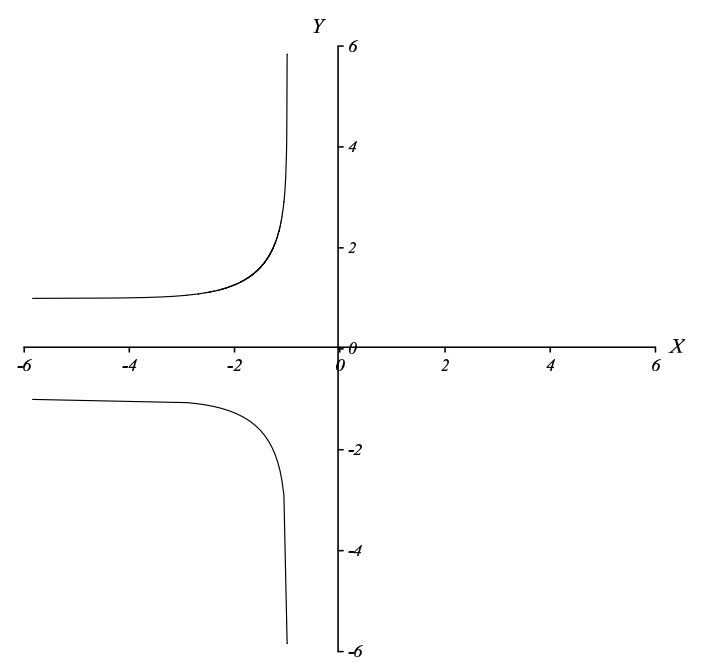

Figure 5: The forme of the free surface

\section{References}

[1] W. Peng and D. F. Parker, An ideal fluid jet impinging on an uneven wall, J. Fluid Mech. No 333 (1997), 231-255

[2] Bloor, Large amptitude surface waves, J. Fluid Mech. № 84 (1978), 167-179

[3] King, A. C., A note on the impact of a jet on a porous wall., IMA J. Appl. Maths., $\mathrm{N}^{\circ} 45$, (2002),139-146

[4] H. Serguine, Numerical resolution of a non-linear problem in a field at free border due to a jet against a infinite vertical plate., Thesis of Magister University of M'sila,(2004),

Received: September 15, 2006 